IJMMS 26:7 (2001) 437-444

PII. S0161171201002307

http://ijmms.hindawi.com

(c) Hindawi Publishing Corp.

\title{
ON THE CORRECT FORMULATION OF A NONLINEAR DIFFERENTIAL EQUATION IN BANACH SPACES
}

\author{
MAHMOUD M. EL-BORAI, OSAMA L. MOUSTAFA, and FAYEZ H. MICHAEL
}

(Received 28 September 1998)

ABSTRACT. We study the existence and uniqueness of the initial value problems in a Banach space $E$ for the abstract nonlinear differential equation $\left(d^{n-1} / d t^{n-1}\right)(d u / d t+$ $A u)=B(t) u+f(t, W(t))$, and consider the correct solution of this problem. We also give an application of the theory of partial differential equations.

2000 Mathematics Subject Classification. 34G20, 47N20.

1. Introduction. Let $E$ be a Banach space. Suppose that $\left\{\left(B_{i}(t), i=1,2, \ldots, v\right), B(t)\right.$, $\left.t \in I=\left[0, T_{0}\right]\right\}$ are families of closed linear operators defined on dense sets $S_{1}, S_{2}, \ldots, S_{v}$, $F$ in $E$, independent of $t$. Let $-A$ be a closed linear operator defined on a dense set $S$ in $E$ such that $S \subset F, S \subset S_{i},(i=1,2, \ldots, v)$.

Suppose that the range of these operators are in $E$, therefore consider the abstract nonlinear differential equation

$$
\begin{gathered}
\frac{d^{n-1}}{d t^{n-1}}\left(\frac{d u}{d t}+A u\right)=B(t) u+f(t, W), \\
\left.u\right|_{t=0}=g_{0},\left.\frac{d u}{d t}\right|_{t=0}=g_{1},\left.\frac{d^{2} u}{d t^{2}}\right|_{t=0}=g_{2}, \ldots,\left.\frac{d^{n-1} u}{d t^{n-1}}\right|_{t=0}=g_{n-1},
\end{gathered}
$$

where all the elements $g_{0}, g_{1}, g_{2}, \ldots, g_{n-1} \in S, W=\left(B_{1}(t) u, B_{2}(t) u, \ldots, B_{v}(t) u\right)$ and $f$ is a given abstract nonlinear function defined on $I \times E^{v}$ with values in $E$. Without loss of generality, we assume that

$$
\left.u\right|_{t=0}=\left.\frac{d u}{d t}\right|_{t=0}=\left.\frac{d^{2}}{d t^{2}}\right|_{t=0}=\cdots=\left.\frac{d^{n-1}}{d t^{n-1}}\right|_{t=0}=\theta,
$$

where $\theta$ is the zero element of the Banach space $E$. Let $f$ be uniformly Hölder continuous for all $t \in I$, that means

$$
\left\|f(t, W)-f\left(t^{*}, W\right)\right\| \leq K\left|t-t^{*}\right|^{\beta},
$$

for all $t$ and $t^{*}$ in $I$ and all $W$ in $E^{v}$, the constant $K$ and $\beta$ are positive and $\beta<1$, where $\|\cdot\|$ is the norm in $E$. For all $W, W^{*} \in E^{v}, W=\left(w_{1}, w_{2}, \ldots, w_{v}\right), W^{*}=\left(w_{1}^{*}, w_{2}^{*}, \ldots, w_{v}^{*}\right)$, and $t \in I$, the function $f$ satisfies the Lipschitz condition

$$
\left\|f(t, W)-f\left(t, W^{*}\right)\right\| \leq K_{1} \sum_{i=1}^{v}\left\|w_{i}-w_{i}^{*}\right\| .
$$

For every $z \in S_{i} \cap F$ the functions $B_{i}(t) z$ and $B(t) z$ are uniformly Hölder continuous 
for $t \in I$ and $i=1,2,3, \ldots, v$ with exponents $\beta^{\prime}$ and $\beta^{\prime \prime}$, respectively-without loss of generality, we can suppose that $\beta=\beta^{\prime}=\beta^{\prime \prime}$. The space of continuous functions $u(t)$ with $t \in I$ and $u(t) \in E$ is denoted by $C^{E}(I)$. The norm in this space is defined by

$$
\|u\|_{C^{E}(I)}=\max _{t \in I}\|u(t)\| .
$$

Suppose that $-A$ generates a semigroup $\{T(t), t \in I\}$ strongly continuous for all $t \geq 0$, this class of semigroup is called $C_{0}$ (see [11, 12]). Furthermore, suppose that $T(t) v \in S$ for all $v \in E, t>0$ (see [6]).

Assume that if there exist $0<\delta<1$ and a positive constant $M$, then

$$
\begin{gathered}
\left\|B\left(t_{2}\right) T\left(t_{1}\right) v\right\| \leq \frac{M}{t_{1}^{\delta}}\|v\|, \\
\left\|B_{i}\left(t_{2}\right) T\left(t_{1}\right) v\right\| \leq \frac{M}{t_{1}^{\delta}}\|v\|,
\end{gathered}
$$

where $M$ is a positive constant and $0<\delta<1$ for all $v \in E, t_{2} \in I, t_{1} \in\left(0, T_{0}\right]$ with $i=1,2, \ldots, v$. In this paper, we prove the existence and the uniqueness of the solution of the Cauchy problem (1.1) and (1.2). The correct formulation of the considered problem is also proved, finally we give an application of the theory of partial differential equations.

2. The solution of the problem. In this section, we discuss the existence and uniqueness of the solution of the initial value problem (1.1) and (1.3). Define on $C^{E}(I)$, a distance function (metric) $\rho$ by

$$
\rho\left(u_{1}, u_{2}\right)=\max _{t \in I} e^{-\lambda t}\left\|u_{1}(t)-u_{2}(t)\right\|,
$$

where $u_{1}, u_{2} \in C^{E}(I), \lambda>1$ being a fixed number. It is clear that $\left(C^{E}(I), \rho\right)$ is a metric space, (see [2, 3]).

THEOREM 2.1. The abstract initial value problem (1.1) and (1.3) has a weak solution in the metric space $\left(C^{E}(I), \rho\right)$ for every $t \in I$.

Proof. From equation (1.1), let

$$
\frac{d u}{d t}=-A u+v
$$

The desired solution $u$ of the above equation can be written in the form (see [1, 11, 12])

$$
u(t)=\int_{0}^{t} T(t-s) v(s) d s
$$

where $v$ satisfies

$$
\begin{gathered}
\frac{d^{n-1} v}{d t^{n-1}}=B(t) \int_{0}^{t} T(t-s) v(s) d s+f(t, W), \\
W=\left(w_{1}, w_{2}, \ldots, w_{v}\right), \quad w_{i}(t)=B_{i}(t) \int_{0}^{t} T(t-s) v(s) d s .
\end{gathered}
$$


Integrating (2.4) $(n-1)$ times, we get

$$
\begin{aligned}
v= & \int_{0}^{t} \int_{s}^{t} \int_{s}^{y_{n-1}} \cdots \int_{s}^{y_{3}} \int_{s}^{y_{2}} B\left(y_{1}\right) T\left(y_{1}-s\right) v(s) d y_{1} d y_{2} \cdots d y_{n-1} d s \\
& +\int_{0}^{t} \int_{0}^{\xi_{n-1}} \cdots \int_{0}^{\xi_{3}} \int_{0}^{\xi_{2}} f\left(\xi_{1}, W\left(\xi_{1}\right)\right) d \xi_{1} d \xi_{2} \cdots d \xi_{n-1} .
\end{aligned}
$$

Let $Q$ be an operator defined on $C^{E}(I)$ by

$$
\begin{aligned}
Q v= & \int_{0}^{t} \int_{s}^{t} \int_{s}^{y_{n-1}} \cdots \int_{s}^{y_{3}} \int_{s}^{y_{2}} B\left(y_{1}\right) T\left(y_{1}-s\right) v(s) d y_{1} d y_{2} \cdots d y_{n-1} d s \\
& +\int_{0}^{t} \int_{0}^{\xi_{n-1}} \cdots \int_{0}^{\xi_{3}} \int_{0}^{\xi_{2}} f\left(\xi_{1}, W\left(\xi_{1}\right)\right) d \xi_{1} d \xi_{2} \cdots d \xi_{n-1} .
\end{aligned}
$$

We prove that $Q$ is a contraction mapping. We notice that

$$
\left\|Q v-Q v^{*}\right\| \leq K_{1} \int_{0}^{t} \int_{s}^{t} \int_{s}^{y_{n-1}} \cdots \int_{s}^{y_{2}}\left(y_{1}-s\right)^{-\delta}\left\|v(s)-v^{*}(s)\right\| d y_{1} \cdots d y_{n-1} d s
$$

which gives

$$
\rho\left(Q v, Q v^{*}\right) \leq \frac{K_{1} T_{0}^{n-\delta} \Gamma(1-\delta)}{\Gamma(n-\delta) \lambda^{n-2}} \rho\left(v, v^{*}\right)
$$

For a sufficiently large $\lambda$ we deduce that $Q$ is a contraction operator therefore there exists a unique fixed point such that (see $[2,3,1]) Q v=v \in C^{E}(I)$, which proves the existence and uniqueness of a weak solution $u$ in $C^{E}(I)$.

We prove that $|f(t, W(t))|$ is bounded on the interval $\left[0, T_{0}\right]$.

THEOREM 2.2. If the function $f(t, W(t))$ satisfies the conditions (1.4) and (1.8), then $|f(t, W(t))|$ is bounded for all $t \in I$.

Proof. From condition (1.4), it is clear that

$$
\begin{aligned}
\|f(t, W(t))-f(t, \theta, \ldots, \theta)\| & =\|f(t, W(t))-f(t, W(0))\| \leq K \sum_{i=1}^{v}\left\|w_{i}(t)\right\| \\
& =K \sum_{i=1}^{v}\left\|\int_{0}^{t} B_{i}(t) T(t-s) v(s) d s\right\| .
\end{aligned}
$$

From (1.8), we get the required result.

TheOREm 2.3 (see $[1,4,11])$. The function $u(t)$ is an element of $S$ for every $t \in I$ and so $u \in C^{S}\left[0, T_{0}\right]$. 
Proof. To prove this theorem, it is enough to show that $v(t)$ satisfies the Lipschitz condition in $t \in I$

$$
\begin{aligned}
v\left(t_{2}\right)-v\left(t_{1}\right)= & \int_{0}^{t_{2}} \int_{t_{1}}^{t_{2}} \int_{s}^{y_{n-1}} \cdots \int_{s}^{y_{3}} \int_{s}^{y_{2}} B\left(y_{1}\right) T\left(y_{1}-s\right) v(s) d y_{1} d y_{2} \cdots d y_{n-1} d s \\
& +\int_{t_{1}}^{t_{2}} \int_{s}^{t_{2}} \int_{s}^{y_{n-1}} \cdots \int_{s}^{y_{3}} \int_{s}^{y_{2}} B\left(y_{1}\right) T\left(y_{1}-s\right) v(s) d y_{1} d y_{2} \cdots d y_{n-1} d s \\
& +\int_{t_{1}}^{t_{2}} \int_{0}^{\xi_{n-1}} \cdots \int_{0}^{\xi_{3}} \int_{0}^{\xi_{2}} f\left(\xi_{1}, W\left(\xi_{1}\right)\right) d \xi_{1} d \xi_{2} \cdots d \xi_{n-1} d s .
\end{aligned}
$$

The theorem is proved by using the above equation and (1.6).

To complete the proof of the existence and uniqueness of the solution (strongly) we prove that each of the following derivative

$$
\frac{d u}{d t}, \frac{d^{2} u}{d t^{2}}, \ldots, \frac{d^{n-1} u}{d t^{n-1}}
$$

belong to $C^{G}(I)$, let $\Psi_{1}(t)=B(t) u(t)$ and $\Psi_{2}(t)=f(t, W(t))$. From (1.1), we can write formally

$$
\begin{aligned}
\frac{d^{r} u(t)}{d t^{r}}= & \int_{0}^{t} \int_{s}^{y_{n-r-1}} \cdots \int_{s}^{y_{3}} \int_{s}^{y_{2}} T\left(y_{1}-s\right) \Psi_{1}(s) d y_{1} d y_{2} \cdots d y_{n-r-1} d s \\
& +\int_{0}^{t} \int_{s}^{\xi_{n-r-1}} \cdots \int_{s}^{\xi_{3}} \int_{s}^{\xi_{2}} T\left(\xi_{1}-s\right) \Psi_{2}(s) d \xi_{1} d \xi_{2} \cdots d \xi_{n-r-1} d s .
\end{aligned}
$$

To get the required result, we must prove that $\Psi_{1}$ and $\Psi_{2}$ satisfies a uniform Hölder condition for $t \in I$. Suppose that $t_{2}>t_{1}$, therefore it is easy to show that

$$
\begin{aligned}
\Psi_{1}\left(t_{2}\right)-\Psi_{1}\left(t_{1}\right)= & \int_{0}^{t_{1}} B\left(t_{2}\right) T\left(t_{1}-s\right)[T(t-t)-J] v(s) d s \\
& +\int_{0}^{t_{1}}\left[B\left(t_{2}\right)-B\left(t_{1}\right)\right] T\left(t_{1}-s\right) v(s) d s \\
& +\int_{t_{1}}^{t_{2}} B\left(t_{2}\right) T(t-s) v(s) d s
\end{aligned}
$$

where $J$ is the identity operator on $E$,

$$
\begin{aligned}
\left\|\Psi_{2}\left(t_{2}\right)-\Psi_{2}\left(t_{1}\right)\right\| & \leq\left\|f\left(t_{2}, W\left(t_{2}\right)\right)-f\left(t_{1}, W\left(t_{2}\right)\right)\right\|+\left\|f\left(t_{1}, W\left(t_{2}\right)\right)-f\left(t_{1}, W\left(t_{1}\right)\right)\right\| \\
& \leq K_{1}\left(t_{2}-t_{1}\right)^{\beta}+K_{2} \sum_{i=1}^{v}\left\|A_{i}\left(t_{2}\right) u\left(t_{2}\right)-A_{i}\left(t_{1}\right) u\left(t_{1}\right)\right\|,
\end{aligned}
$$

where $K_{1}$ and $K_{2}$ are positive constants. Similarly, as in [9], we can prove that $\Psi_{1}$ and $\Psi_{2}$ satisfies Hölder condition in $t \in I$, therefore $(d u / d t) \in C^{S}(I)$ and $(d v / d t)$ is continuous for all $t \in I$.

Now, $A u(t)$ can be written in the form

$$
A u(t)=\int_{0}^{t} \int_{s}^{t} \int_{s}^{y_{n-1}} \cdots \int_{s}^{y_{3}} \int_{s}^{y_{2}} A T\left(y_{1}-s\right)\left[\Psi_{1}(s)+\Psi_{2}(s)\right] d y_{1} d y_{2} \cdots d y_{n-1} d s .
$$


Thus differentiate $(n-1)$ times we get

$$
\frac{d^{n-1}}{d t^{n-1}}[A u]=\int_{0}^{t} A T\left(y_{1}-s\right)\left[\Psi_{1}(s)+\Psi_{2}(s)\right] d s=A \frac{d^{n-1} u}{d t^{n-1}} .
$$

Therefore,

$$
\frac{d^{n} u}{d t^{n}}=\frac{d^{n-1} v}{d t^{n-1}}-A \frac{d^{n-1} u}{d t^{n-1}}
$$

is continuous on $I$. Consequently,

$$
u(t)=\int_{0}^{t} T(t-s) v(s) d s
$$

represent the unique solution of the considered Cauchy problem (compare with [8, $10,11])$.

3. Correct solution. In this section, we prove the correct formulation of the considered initial value problem (1.1) and (1.2). In other words, we prove the continuous dependent of the solution of the problem on the initial conditions. Let $\left\{u^{m}\right\}$ be a sequence of solutions of the initial value problem

$$
\begin{gathered}
\frac{d^{n} u^{m}}{d t^{n}}+A \frac{d^{n-1} u^{m}}{d t^{n-1}}=B(t) u^{m}+f\left(t, W^{m}\right), \\
\left.u^{m}\right|_{t=0}=g_{0}^{m} \in S,\left.\frac{d u^{m}}{d t}\right|_{t=0}=g_{1}^{m}, \ldots,\left.\frac{d^{n-1} u^{m}}{d t^{n-1}}\right|_{t=0}=g_{n-1}^{m},
\end{gathered}
$$

where $W^{m}$ is the sequence $W^{m}=\left(B_{1}(t) u^{m}, B_{2}(t) u^{m}, \ldots, B_{v}(t) u^{m}\right)$.

Theorem 3.1 (see $[7,9]$ ). Let the sequences $\left\{g_{0}^{m}\right\},\left\{g_{1}^{m}\right\},\left\{g_{2}^{m}\right\}, \ldots,\left\{g_{n-1}^{m}\right\},\left\{A g_{n-1}^{m}\right\}$, be convergent in $E$ to $g_{0}, g_{1}, g_{2}, \ldots, g_{n-1}, A g_{n-1}$, respectively. If the sequences $\left\{B(t) g_{0}^{m}\right\}$, $\left\{B(t) g_{1}^{m}\right\},\left\{B(t) g_{2}^{m}\right\}, \ldots,\left\{B(t) g_{n-1}^{m}\right\},\left\{B_{i}(t) g_{0}^{m}\right\},\left\{B_{i}(t) g_{1}^{m}\right\},\left\{B_{i}(t) g_{2}^{m}\right\}, \ldots,\left\{B_{i}(t) g_{n-1}^{m}\right\}$ are uniformly convergent with respect to $t \in I$ in $E$ to $B(t) g_{0}, B(t) g_{1}, B(t) g_{2}, \ldots$, $B(t) g_{n-1}, B_{i}(t) g_{0}, B_{i}(t) g_{1}, B_{i}(t) g_{2}, \ldots, B_{i}(t) g_{n-1}, \quad i=1,2, \ldots, v$, respectively, where $g_{0}, g_{1}, g_{2}, \ldots, g_{n-1}$ are elements in $G$, then the sequence of solutions $\left\{u^{m}(t)\right\}$ of the problem (3.1) and (3.2) converges in the metric space $C^{E}(I)$ to the solution $u(t)$ of the considered problem with the initial conditions,

$$
\left.u\right|_{t=0}=g_{0},\left.\frac{d u}{d t}\right|_{t=0}=g_{1}, \ldots, \frac{d^{n-1} u}{d t^{n-1}}=g_{n-1},
$$

Proof. Let

$$
\zeta^{m}(t)=u^{m}(t)-\sum_{k=0}^{n-1} \frac{t^{k}}{k !} g_{k}^{m}
$$

substitute in equation (1.8), we get

$$
\frac{d^{n} \zeta^{m}}{d t^{n}}+A \frac{d^{n-1} \zeta^{m}}{d t^{n-1}}=B(t) \zeta^{m}+f^{*}\left(t, W^{m}\right)
$$


with the initial conditions

$$
\left.\zeta^{m}\right|_{t=0}=0,\left.\frac{d \zeta^{m}}{d t}\right|_{t=0}=0, \ldots,\left.\frac{d^{n-1} \zeta^{m}}{d t^{n-1}}\right|_{t=0}=0
$$

where

$$
\begin{aligned}
f^{*}\left(t, W^{m}\right) & =\sum_{k=0}^{n-1}\left(B \frac{t^{k}}{k !} g_{k}^{m}\right)+f\left(t, W^{m}\right)-A g_{n-1}^{m}, \\
W^{m} & =\left(B_{1}(t) u^{m}, B_{2}(t) u^{m}, \ldots, B_{v}(t) u^{m}\right) \\
& =\left(B_{1} \zeta^{m}+\sum_{k=0}^{n-1} B_{1}(t) \frac{t^{k}}{k !} g_{k}^{m}, \ldots, B_{v}(t) \zeta^{m}+\sum_{k=0}^{n-1} B_{v}(t) \frac{t^{k}}{k !} g_{k}^{m}\right) .
\end{aligned}
$$

Set

$$
\frac{d \zeta^{m}(t)}{d t}+A \zeta^{m}(t)=P^{m}(t)
$$

therefore,

$$
\frac{d^{n-1}}{d t^{n-1}}\left(P^{m}(t)\right)=B(t) \zeta^{m}(t)+f^{*}\left(t, W^{m}\right)
$$

It is clear that

$$
\begin{aligned}
P^{m}(t)= & \int_{0}^{t} \int_{s}^{t} \int_{s}^{y_{n-1}} \cdots \int_{s}^{y_{3}} \int_{s}^{y_{2}} B\left(y_{1}\right) T\left(y_{1}-s\right) P^{m}(s) d y_{1} d y_{2} \cdots d y_{n-1} d s \\
& +\int_{0}^{t} \int_{0}^{\xi_{n-1}} \cdots \int_{0}^{\xi_{3}} \int_{0}^{\xi_{2}} f^{*}\left(\xi_{1}, W^{m}\left(\xi_{1}\right)\right) d \xi_{1} d \xi_{2} \cdots d \xi_{n-1},
\end{aligned}
$$

we can easily deduce that

$$
\begin{gathered}
\left\|P^{m}(t)-P^{r}(t)\right\| \\
\leq \int_{0}^{t} \int_{s}^{t} \int_{s}^{y_{n-1}} \cdots \int_{s}^{y_{3}} \int_{s}^{y_{2}} \\
+\int_{0}^{t} \int_{0}^{\xi_{n-1}} \cdots \int_{0}^{\xi_{3}} \int_{0}^{\xi_{2}}\left[\sum_{k=0}^{n-1} B \frac{t^{k}}{k !}\left\|g_{k}^{m}-g_{k}^{r}\right\|\right. \\
\left.+\left\|f\left(t, W^{m}\right)-f\left(t, W^{r}\right)\right\|+A\left\|\left(g_{n-1}^{m}-g_{n-1}\right)\right\|\right] \\
\times d \xi_{1} d \xi_{2} \cdots d \xi_{n-1} .
\end{gathered}
$$

Multiply by $e^{-\lambda t}$ and using the metric defined by equation (2.1), we get

$$
\begin{aligned}
\rho\left(P^{m}, P^{r}\right) \leq & K \rho\left(A g_{n-1}^{m}, A g_{n-1}^{r}\right)+K\left[\sum_{k=0}^{n-1} \rho\left(B g_{k}^{m}, B g_{k}^{r}\right)\right] \\
& +K \sum_{k=1}^{v} \sum_{j=0}^{n-1} \rho\left(A_{k} g_{j}^{m}, A_{k} \mathcal{g}_{j}^{r}\right) .
\end{aligned}
$$

According to all the conditions before, the sequence $\left\{P^{m}\right\}$ is fundamental and hence converges to $P$ in $C^{E}(I)$. But

$$
\zeta^{m}(t)=\int_{0}^{t} T(t-s) P^{m}(s) d s .
$$


Therefore, the sequence $\left\{u^{m}(t)\right\}$ uniformly converges with respect to $t \in I$ in $E$ to the required solution (compare with $[6,5,4,9]$ ).

4. Application. Consider the Cauchy problem

$$
\frac{\partial^{n-1}}{\partial t^{n-1}}(L u)=\sum_{|\alpha| \leq 2 m-1} a_{\alpha}(x, t) D^{\alpha} u+f(x, t, W),
$$

with the initial conditions

$$
\left.u(x, t)\right|_{t=0}=g_{0}(x),\left.\frac{\partial u(x, t)}{\partial t}\right|_{t=0}=g_{1}(x), \ldots,\left.\frac{\partial^{n-1} u(x, t)}{\partial t^{n-1}}\right|_{t=0}=g_{n-1}(x),
$$

where

$$
\begin{gathered}
L u=\frac{\partial u}{\partial t}+A(x, D) u, \quad A(x, D)=\sum_{|\alpha| \leq 2 m} b_{\alpha}(x) D^{\alpha}, \\
W=\left(B_{1}(t) u, B_{2}(t) u, \ldots, B_{v}(t) u\right), \quad B_{i}(t)=\sum_{|\alpha| \leq 2 m-1} C_{\alpha, i}(x, t) D^{\alpha},
\end{gathered}
$$

for $x=\left(x_{1}, x_{2}, \ldots, x_{n}\right) \in \mathbb{R}^{n}, D_{i}=\partial / \partial x_{i}, D^{\alpha}=D_{1}^{\alpha_{1}} D_{2}^{\alpha_{2}} \cdots D_{n}^{\alpha_{n}}, \alpha=\left(\alpha_{1}, \alpha_{2}, \ldots, \alpha_{n}\right)$ is an $n$-dimensional multi-index, and $|\alpha|=\alpha_{1}+\alpha_{2}+\cdots+\alpha_{n}$. Let $M$ be an open set in the $n$-dimensional Euclidean space $\mathbb{R}^{n}$, and let $L_{2}(M)$ be the space of all square integrable functions on $M$. We denote by $C^{m}(M)$ the set of all continuous real-valued functions in $M$ together with all their $m$-partial derivatives, and we denote by $C_{0}^{m}(M)$ the subset of $C^{m}(M)$ consisting of all functions having a compact support. Let $H^{m}(M)$ be the complete space of $C^{m}(M)$ with respect to the norm (see [2, 3])

$$
\|f\|_{m}=\left[\sum_{|\alpha| \leq m} \int_{M}\left|D^{\alpha} f(x)\right|^{2} d x\right]^{1 / 2} .
$$

For any $0<b<\infty$ denote by $\Omega_{b}$ the cylinder $\{(x, t): x \in M, 0<t<b\}$, and by $\Gamma_{b}$ the boundary $\{(x, t): x \in \partial M, 0<t<b\}$, where $\partial M$ is the boundary of $M$. We say that $L$ is uniformly parabolic in $\bar{M}$, the closure of $M$ if the coefficients $b_{\alpha}$ are continuous on $\bar{M}$ and if

$$
(-1)^{m} \sum_{|\alpha|=2 m} b_{\alpha}(x) \eta^{\alpha} \geq C|\eta|^{2 m}, \quad c>0
$$

for all $x \in M$ and for all $\eta \in R^{n}$, where $\eta^{\alpha}=\eta_{1}^{\alpha_{1}} \eta_{2}^{\alpha_{2}} \cdots \eta_{n}^{\alpha_{n}}$, and $|\eta|^{2}=\eta_{1}^{2}+\eta_{2}^{2}+\cdots+$ $\eta_{n}^{2}$. Suppose also that the coefficients $a_{\alpha}, C_{\alpha, i}$ are continuous on $\Omega_{b}$ and satisfies a uniform Hölder condition in $t \in[0, b]$. The Cauchy problem (4.1) and (4.2) can be written in the abstract form (1.1) and (1.2), where $A$ is the operator with domain $S=H^{2 m}(M) \cap H_{0}^{m}(M)$ given by

$$
A u=A(x, D) u=\sum_{|\alpha| \leq 2 m} b_{\alpha}(x) D^{\alpha} u .
$$

Let $E=L_{2}(M)$. Then the domain $S$ is dense in $L_{2}(M)$. The operators $B(t), B_{1}(t), B_{2}(t)$, $\ldots, B_{v}(t)$ are given by

$$
B(t)=\sum_{|\alpha| \leq 2 m-1} a_{\alpha}(x, t) D^{\alpha}, \quad B_{i}(t)=\sum_{|\alpha| \leq 2 m-1} C_{\alpha, i}(x, t) D^{\alpha},
$$


where $i=1,2, \ldots, v$. The domain of these operators can be taken $H^{2 m-1}(M) \cap H_{0}^{m}(M)$ which is dense in $L_{2}(M)$ (see $[1,2,10]$ ). Therefore, we can assume that

$$
S_{1}=S_{2}=\cdots=S_{v}=F=H^{2 m-1}(M) \cap H_{0}^{m}(M) .
$$

Suppose that $g_{0}(x), g_{1}(x), \ldots, g_{n-1}(x)$ are given functions in $S$. Since $L u$ is uniformly parabolic, it follows that $-A=-A(x, D)$ generates a semi-group $\{T(t)\}$ of class $C_{0}$. It can be proved that $T(t)$ satisfies the condition (1.7) and (1.8). Consequently, [2, 6] can be applied to the Cauchy problem (4.1) and (4.2). This means that the considered problem can be solved in $S$ without any restrictions on the characteristic forms of the operators

$$
\sum_{|\alpha| \leq 2 m} a_{\alpha}(x, t) D^{\alpha}, \quad \sum_{|\alpha| \leq 2 m} C_{\alpha, i}(x, t) D^{\alpha},
$$

which depends only on the continuity of the functions $g_{0}, g_{1}, \ldots, g_{n-1}$.

\section{REFERENCES}

[1] H. A. S. Abujabal and M. M. El-Borai, On the Cauchy problem for some abstract nonlinear differential equations, Korean J. Comput. Appl. Math. 3 (1996), no. 2, 279-290. MR 97g:34078.

[2] R. A. Adams, Sobolev Spaces, Pure and Applied Mathematics, vol. 65, Academic Press, New York, 1975. MR 56\#9247. Zbl 314.46030.

[3] R. E. Edwards, Functional Analysis. Theory and Applications, Holt, Rinehart and Winston, New York, 1965. MR 36\#4308. Zbl 182.16101.

[4] M. M. El-Borai, On the correct formulation of the Cauchy problem, Vestnik Moskov. Univ. Ser. I Mat. Meh. 23 (1968), no. 4, 15-21 (Russian). MR 40\#4597. Zbl 157.16703.

[5] __ Some characterization of an abstract differential equation, Proc. Math. Phys. Soc. Egypt (1979), no. 48, 15-23. MR 81h:34069. Zbl 448.47026.

[6] - On the initial value problem for a partial differential equation with operator coefficients, Int. J. Math. Math. Sci. 3 (1980), no. 1, 103-111. MR 82b:35148. Zbl 457.35086 .

[7] E. Hille and R. S. Phillips, Functional Analysis and Semi-groups, revised ed., American Mathematical Society Colloquium Publications, vol. 31, American Mathematical Society, Rhode Island, 1957. MR 19,664d. Zbl 078.10004.

[8] T. Kato, Nonlinear semigroups and evolution equations, J. Math. Soc. Japan 19 (1967), 508-520. MR 37\#1820. Zbl 163.38303.

[9] T. Kato and H. Tanabe, On the abstract evolution equation, Osaka Math. J. 14 (1962), 107-133. MR 25\#4367. Zbl 106.09302.

[10] R. H. Martin, Jr., Nonlinear Operators and Differential Equations in Banach Spaces, Pure and Applied Mathematics, John Wiley \& Sons, New York, 1976. MR 58\#11753. Zbl 333.47023.

[11] S. M. Rankin, III, Semilinear evolution equations in Banach spaces with application to parabolic partial differential equations, Trans. Amer. Math. Soc. 336 (1993), no. 2, 523-535. MR 93f:34105. Zbl 785.34044.

[12] H. Tanabe, Equations of Evolution, Monographs and Studies in Mathematics, vol. 6, Pitman, London, 1979, translated from the Japanese by N. Mugibayashi and H. Haneda. MR 82g:47032. Zbl 417.35003.

Mahmoud M. EL-Borai, Osama L. Moustafa, and Fayez H. Michael: Faculty of Science, ALEXANDRIA UNIVERSITY, ALEXANDRIA, EGYPT 


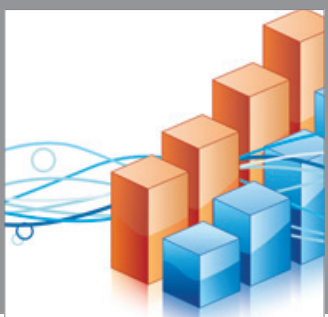

Advances in

Operations Research

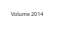

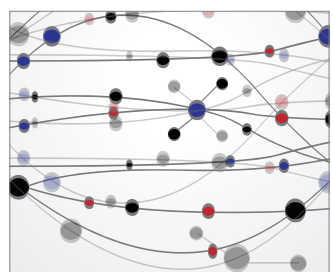

\section{The Scientific} World Journal
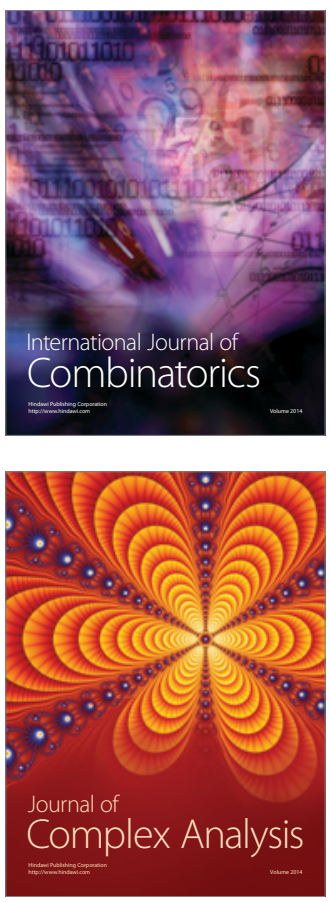

International Journal of

Mathematics and

Mathematical

Sciences
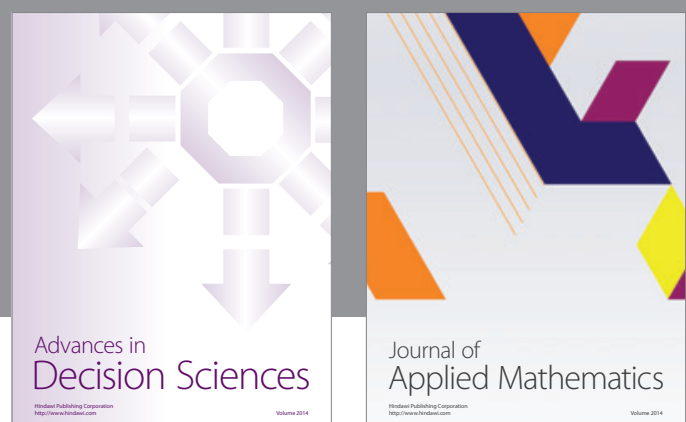

Journal of

Applied Mathematics
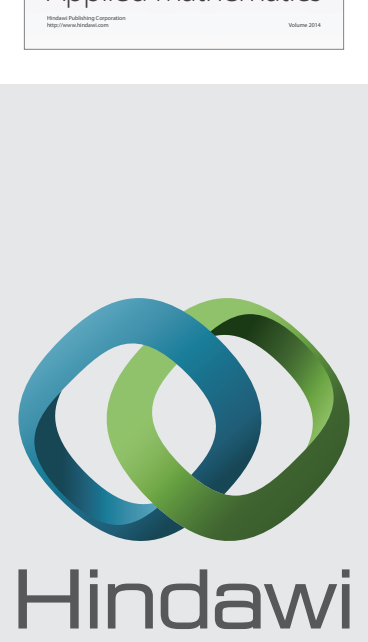

Submit your manuscripts at http://www.hindawi.com
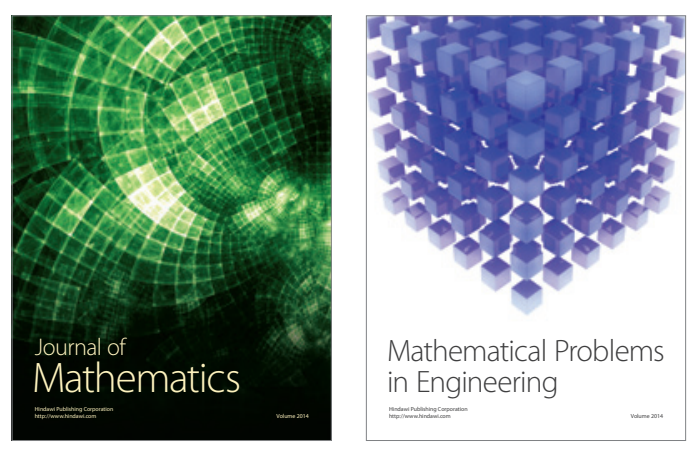

Mathematical Problems in Engineering
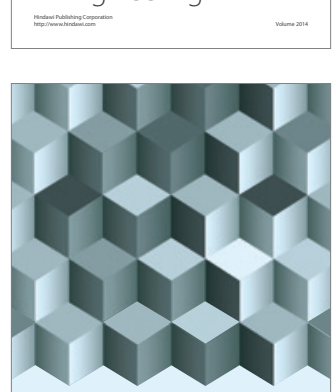

Journal of

Function Spaces
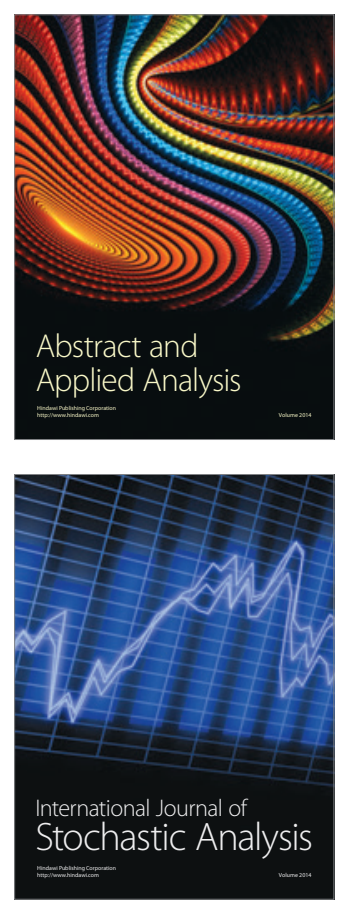

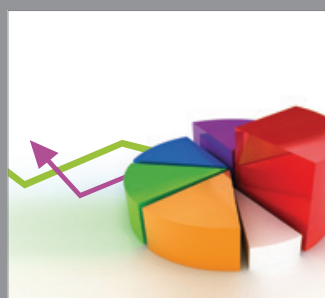

ournal of

Probability and Statistics

Promensencen
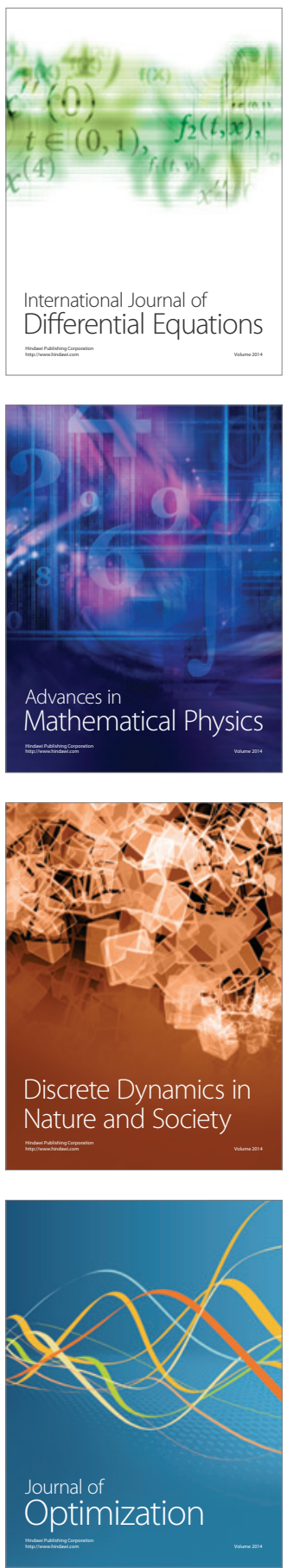